\title{
QUÉ PUEDE DECIR LA NEUROCIENCIA SOBRE EL LIBRE ALBEDRÍO: CUESTIONANDO SU METODOLOGÍA Y LA POSIBILIDAD DE RESOLVER EL PROBLEMA
}

\author{
ANDRÉS D. RICHART \\ Universidad de Valencia
}

\begin{abstract}
RESUMEN: El antiguo problema de la libertad y el determinismo ha sido retomado en nuestros días por las neurociencias desde una perspectiva naturalista. Son muchos los problemas metodológicos y conceptuales a los que se enfrentan los investigadores al tratar de resolver científicamente la pregunta por la existencia del libre albedrío. Nuestro objetivo será someter a crítica uno de los presupuestos de estas investigaciones, la posibilidad de cuantificar la libertad como un hecho. Para ello expondremos en primer lugar las dificultades relativas a esta pretensión. Tras ello estableceremos cuáles son las condiciones de posibilidad de la libertad en aras de someter a crítica las perspectivas psicológica y neurocientífica, sugerimos que ambas son incapaces de ofrecer conocimiento sobre la existencia de tales condiciones. Asimismo, ofreceremos algunas consideraciones metodológicas tomando como eje de la crítica el famoso experimento de Libet.
\end{abstract}

PALABRAS CLAVE: determinismo; libre albedrío; naturalismo; neurociencia.

\section{What neuroscience can tell us about free will: questioning its methodology and its possibility to solve the problem}

ABSTRACT: The old problem of free will and determinism has been taken up today by the neurosciences from a naturalistic perspective. There are many methodological and conceptual problems that researchers face in trying to scientifically solve the question of the existence of free will. Our goal will be subject to review one of the budgets of these investigations, the possibility of quantifying free will as a fact. To do this we will discuss first the difficulties relating to this claim. After that we will establish what are the conditions of possibility of free will in order to criticize the psychological and neuroscientific perspectives, we suggest that both are unable to offer knowledge about the existence of these conditions. Also, we offer some methodological considerations attending the famous Libet's experiment.

KEY WORDS: determinism; free will; naturalism; neuroscience.

\section{INTRODUCCIÓN}

Con el auge de las neurociencias ha nacido la pretensión de investigar de forma naturalista, mediante el estudio del cerebro, si existe el libre albedrío o si por el contrario el ser humano está determinado de forma unívoca en su conducta. La libertad es una de las condiciones necesarias para que una acción sea genuinamente moral. La posibilidad de actuar de diferentes formas y de poder escoger entre ellas es condición indispensable para la moralidad, si la conducta estuviese determinada por una cadena causal en la que se imbricasen nuestra biología, nuestro entorno y todo el devenir del universo en un sentido más amplio y holístico, entonces formaríamos parte de una complejísima red en la que unos hechos conducen a otros de forma unívoca, incluyendo nuestra conducta. El sentimiento de libertad, en tal caso, no sería más que una ilusión. 
Si se demostrase que esto es así conceptos como el de responsabilidad y el de elección, y toda nuestra concepción no solo de la moral y la ética, sino de la realidad humana, del derecho, de la sociología, de la historia, etc., se verían comprometidos, y habrían de ser revisados. Sin embargo, si se demostrase que existe el libre albedrío, podríamos desembarazarnos de estas preocupaciones y continuar con nuestra concepción del ser humano como un ser libre ${ }^{1}$.

Considero que existe una imposibilidad en las ciencias empíricas, y más concretamente en las neurociencias, para resolver la cuestión de si el ser humano puede actuar de forma libre o si se halla determinado de forma unívoca en su conducta. Para respaldar esta tesis pretendo poner en tela de juicio las pretensiones neurocientíficas que defienden la posibilidad de cuantificar la libertad como un hecho. Para ello en primer lugar expondré los principales problemas y cuestiones relativos a la libertad y su cuantificación desde las ciencias empíricas. En segundo lugar, atendiendo a Evers y Cortina estableceré cuáles son las condiciones de posibilidad de la libertad, en aras de aclarar si es posible descubrir la existencia de tales condiciones desde la perspectiva naturalista de las ciencias empíricas y, concretamente, de las neurociencias. Tras ello atenderemos a Aranguren, para poner de relieve cómo una perspectiva meramente psicológica de la libertad no puede ofrecer conclusiones acerca de si la conducta se configura unívocamente o si, por el contrario, permite diversas acciones ante un mismo estímulo. Seguidamente someteremos a crítica la investigación naturalista de las neurociencias en torno a la cuestión de la libertad, centrando el análisis en el experimento de Libet, que tomaremos como eje de la reflexión, prestando especial atención a la crítica que Cortina vierte sobre este. Atenderemos también a Habermas en este punto, cuyas críticas al experimento quedan recogidas por Cortina. Finalmente se plantearán las conclusiones derivadas de todo lo expuesto.

\section{1. ¿Puede cuantificarse la libertad como un hecho?}

La existencia de sujetos libres precisa que estos hayan alcanzado cierto umbral de desarrollado y complejidad cognitiva ${ }^{2}$. Es preciso que se dé la

\footnotetext{
1 CoRtina, A., Neuroética y neuropolítica: Sugerencias para la educación moral, Tecnos, Madrid, 2012, pp. 151-153.

FARAH, M. J., «Neuroethics: The Ethical, Legal, and Societal Impact of Neuroscience» en: Annual Review of Psychology, 63 (2012) 571-91.

Roskies, A. L., «How Does Neuroscience Affect Our Conception of Volition?» en: Annual Review of Neuroscience, 33 (2010) 109-130.

2 Si bien Ayala no plantea esta idea explícitamente, su propuesta en torno a las condiciones de posibilidad de una conducta ética sugiere que el nivel de desarrollo cognitivo está asociado a la posibilidad de emergencia de tales conductas, siendo una de sus condiciones de posibilidad el libre albedrío. Ayala, F. J., «Las raíces biológicas de la moral», en: Ayala, F. J., La evolución de un evolucionista, Universidad de Valencia, Valencia, 2006, pp. 351-369.
} 
existencia de estructuras cerebrales capaces de romper las cadenas causales de nuestra biología o bien capaces de generar una contingencia de consecuencias ante una misma causa ${ }^{3}$, siendo además el sujeto quien puede escoger por autodeterminación una de las opciones disponibles ${ }^{4}$. Sobre las condiciones de posibilidad de la libertad volveremos más adelante. Ahora bien, no sabemos si la evolución ha forjado seres realmente libres, o si más bien ha generado seres que se autoperciben como libres ${ }^{5}$, es precisamente esta cuestión la que las neurociencias pretenden resolver.

Ya que la existencia o inexistencia del libre albedrío presenta como base las estructuras cerebrales que configuran la decisión y la acción, pueden comprenderse las tentativas neurocientíficas de cuantificar la libertad o su inexistencia como hecho neurobiológico. Ahora bien, nada garantiza que la ciencia empírica sea capaz de ofrecer resultados significativos al respecto. La dificultad de llevar a cabo una investigación sobre la existencia del libre albedrío desde una perspectiva naturalista radica en determinar si es o no posible la cuantificación de la libertad o de su inexistencia como un hecho, y de serlo qué consideraciones metodológicas habrían de ser tenidas en cuenta. La neurociencia plantea, con el experimento de Libet como uno de sus estandartes, por otra parte muy susceptible de crítica, la posibilidad de desvelar la respuesta a la cuestión de si estamos determinados o somos libres. ¿Pero está realmente la neurociencia capacitada para ofrecer una respuesta a una cuestión de este tipo? ¿Puede ser la existencia o la inexistencia del libre albedrío cuantificada como un hecho observable?

A lo largo del artículo argumentaré a favor de la siguiente tesis: si bien la existencia o inexistencia del libre albedrío en el ser humano es una realidad, existen dificultades, quizás una imposibilidad, para cuantificar esta desde el método científico, lo cual otorga un papel preminente a la filosofía a la hora de abordar la cuestión. Los experimentos llevados a cabo hasta la fecha no son capaces de ofrecer conclusiones claras al respecto. A pesar de que el método

3 Evers, K., Neuroética. Cuando la materia se despierta, Katz Editores, Madrid, 2010, pp. 86-97.

4 Evers, K., O.c.

Contina, A., Neuroética y neuropolítica: Sugerencias para la educación moral, Tecnos, Madrid, 2012, p. 161.

5 Esta autopercepción ilusoria podría deber su origen al hecho de ofrecer una ventaja evolutiva a la hora de regular la conducta, o bien gracias a ser una consecuencia no prevista por la evolución (by-product) resultante de la concurrencia de ciertas facultades mentales y de usos no previstos por la evolución de estructuras cerebrales desarrolladas para otras funciones.

FARAH, M. J., «Neuroethics: The Ethical, Legal, and Societal Impact of Neuroscience» en: Annual Review of Psychology, 63 (2012) 571-91.

Gould, S. J. y Lewontin, R. C., «The Spandrels of San Marco and the Panglossian Paradigm: A Critique of the Adaptationist Programme», The Royal Society of London, Series B, Vol. 205, $\mathrm{n}^{\circ} .1161$ (1979) 581-598.

Gould, S. J. y VRBa, E. S., «Exaptation - A Missing Term in The Science of Form», en Paleobiology, Vol. 8, no 1 (1982) 4-15. 
científico tiene entre sus competencias explicar y describir la realidad —así como promover y diseñar la aplicación técnica y tecnológica que permite ese conocimiento- eso no significa necesariamente que la ciencia sea capaz de conocer toda la realidad existente. Simplemente algunos aspectos de lo real escapan por completo a los sistemas de medición con los que la ciencia cuenta. Es posible que la investigación en torno a la libertad se enfrente a una realidad de este tipo. Es decir, se ha de considerar la posibilidad de que la mera cuantificación de la actividad y estructuras cerebrales, que subyacen bajo las conductas susceptibles de libertad, sean constitutivamente insuficientes para desvelar si de hecho podemos obrar con libre albedrío.

\section{LAS CONDICIONES DE POSIBILIDAD DE LA LIBERTAD}

Antes de someter a análisis y crítica la posibilidad de cuantificar el libre albedrío desde las ciencias empíricas es preciso establecer cuáles son las condiciones de posibilidad de una conducta realmente libre. Una vez establecidas estas condiciones estaremos en posición de analizar si la perspectiva naturalista de las ciencias empíricas es capaz de resolver la cuestión desde su método. Recurriremos a Evers y Cortina para señalar cuáles son las condiciones de posibilidad necesarias y suficientes para que una conducta pueda ser considerada como libre.

Kathinka Evers en Neuroética: Cuando la materia se despierta aborda la cuestión de la libertad en un capítulo titulado «El cerebro responsable, el libre albedrío y la responsabilidad personal a la luz de las neurociencias ${ }^{6}$. A lo largo del capítulo Evers expone los términos generales del debate libertaddeterminismo para posicionarse en ese debate y exponer su postura respecto a la libertad y la responsabilidad en el ámbito de las neurociencias. Sostiene que el libre albedrío es una estructura neuronal fundamental, junto a otras como el espacio, el tiempo o la causalidad. Con independencia de si la experiencia de libertad es una mera ilusión, Evers sostiene que podemos hablar de actos libres en el sentido de voluntarios, aunque fuesen una construcción causalmente determinada por procesos neuronales no conscientes. Además suscribe la idea de que puede existir una causalidad contingente compatible con la libertad de elección, según la cual ante una misma causa pueden darse dos consecuencias diferentes, entre las cuales la elección sería posible. El teorema de la variabilidad en neurociencias supone un apoyo empírico para tesis de este tipo. ${ }^{7}$ Asimismo considera que se puede ser responsable a pesar de los procesos no conscientes que subyacen a las decisiones y a las acciones, ya que somos personalmente responsables de esos estados y procesos en la medida en que podemos influir

\footnotetext{
6 Evers, K., Neuroética. Cuando la materia se despierta, Katz Editores, Madrid, 2010, pp. 74-112. pp. 86-97.

7 Evers, K., Neuroética. Cuando la materia se despierta, Katz Editores, Madrid, 2010,
} 
en ellos. A lo largo de su reflexión ofrece dos condiciones que han de darse para que un sujeto sea libre:

A. En primer lugar, a la hora de actuar, el sujeto ha de poder producir dos conductas diferentes ante una misma situación, o dicho de otro modo, ha de poder generar dos consecuencias diferentes ante una misma causa. Es lo que Evers considera como una contingencia causal, pueden producirse dos mundos posibles a partir de una causa o conjunto de causas, de tal modo que aun sin escapar de la cadena de causalidad existe la posibilidad de bifurcación dentro de la misma.

B. Esa contingencia de la acción no debe producirse al azar ni por indeterminación, sino de forma autodeterminada. La libertad es autodeterminación, el sujeto ha de poder determinar qué acción llevar a cabo ante la opción de ejecutar varios cursos de acción posibles. Entran en juego aquí la reflexión, las razones y la decisión consciente, qué papel juega cada uno de estos elementos es planteado de diferente modo según diferentes autores.

Esas dos condiciones no se producen solo en el ámbito de lo mental, dado que lo mental arraiga en las estructuras biológicas y cerebrales del sujeto dependerá de su arquitectura cerebral la posibilidad de generar un espacio de decisión entre al menos dos posibles cursos de acción y que además esa decisión se produzca de forma autodeterminada.

Cortina se ocupa también de la cuestión de la libertad. Esta, indica, suele entenderse como una propiedad de la voluntad, y precisa de tres condiciones ${ }^{8}$ :

a) Entre un conjunto de posibilidades la que elegimos está en nuestras manos.

b) La fuente de nuestras acciones está en nosotros, no en algo sobre lo que no tenemos control.

c) Podríamos haber actuado de manera diferente, había alternativas y la posibilidad real de optar por ellas.

La condición $a$ de Evers aglutina las condiciones 1 y 3 de Cortina, mientras que la condición $b$ corresponde con la 2 , respectivamente. Podemos por tanto identificar una coincidencia en la postura de ambas pensadoras. ${ }^{9}$

Las condiciones de posibilidad de una conducta libre - a las que referiremos como $L 1$ y $L 2$ durante la exposición - son, por tanto, las siguientes: L1) dadas

CoRTina, A., o.c., pp. 155-156.

9 Añade Cortina que en caso de que la libertad no existiese y que científicamente pudiese demostrarse, hay quien plantea un compatibilismo entre ese conocimiento y el vivir como si la libertad existiese, aduciendo que es como mejor sabemos organizar nuestra vida. ¿Pero acaso no sería esto ignorar un conocimiento al que aspiramos para mejorar nuestras vidas? Si la libertad no existiese algunas disciplinas como el derecho deberían ser reestructuradas de forma profunda, afirma la autora. 
unas condiciones ha de existir la posibilidad real de escoger entre dos cursos de acción diferentes, y L2) la elección se ha de dar por autodeterminación, no determinadamente, por azar o por indeterminación. Estas condiciones a su vez implican una serie de presupuestos que toda investigación científica debería de resolver. Desglosar estas dos condiciones en los siguientes presupuestos consiste, en realidad, en confeccionar una lista más específica de condiciones de posibilidad, que aparecen planteadas por $L 1$ y $L 2$ tan solo de forma implícita. Entre los presupuestos de $L 1$-referidos como $L 1 a$ y $L 1 b$ - se encuentran los siguientes: L1a) Existe bien una fisura en la cadena causal, o bien una bifurcación posible en la misma (contingencia causal); L1b) existe la posibilidad, brindada por nuestro cerebro, bien de generar una causa que produzca una decisión y una acción (el yo sería esta causa, ver $L 2 b$ ), bien de dirigir la conducta hacia la opción escogida en una contingencia causal, respectivamente. Por su parte entre los presupuestos de $L 2$-referidos como $L 2 a$ y $L 2 b$ - encontramos los siguientes: L2a) Existe un yo, que sería el decisor y el actor; L2b) el yo tiene la capacidad de operar como causa en la decisión y en la acción, bien como una nueva causa dada una fisura en la cadena causal, bien como causa de la elección entre las opciones posibles en una contingencia causal. Así las dos condiciones de posibilidad del libre albedrío expuestas por Evers y Cortina ( $L 1$ y $L 2)$ revelan un total de cuatro condiciones de posibilidad ( $L 1 a, L 1 b, L 2 a$ y $L 2 b)$, que quedan especificadas al desglosar los presupuestos de aquellas dos.

Una investigación naturalista que pretenda argumentar a favor de la existencia o inexistencia del libre albedrío tiene que poder demostrar que todas estas condiciones se dan o no se dan. Serán insuficientes todas aquellas propuestas que pretendan argumentar a favor del determinismo simplemente por haber sido incapaces de demostrar la existencia de la libertad, a tales propuestas se les ha de exigir que demuestren su inexistencia, si es que es posible hacer tal cosa. De igual modo serán insuficientes aquellas propuestas que se posiciones a favor de la existencia del libre albedrío sin haber logrado demostrar que se cumplan todas las condiciones de posibilidad de su existencia.

En los próximos apartados atenderemos a dos ejemplos de propuesta científica que cometen el error señalado, no ofrecen una demostración del cumplimiento o incumplimiento de las condiciones de posibilidad del libre albedrío al afirmar su existencia o su inexistencia, respectivamente. El primer caso corresponde a la perspectiva psicológica ofrecida por Aranguren en su Ética, quien sugiere la existencia del libre albedrío. El segundo caso atiende a la propuesta de Libet basada en sus famosos experimentos neurocientíficos, en virtud de los cuáles se plantea un determinismo conductual que implica la inexistencia del libre albedrío. El análisis de estos dos casos permitirá cumplir dos objetivos. El primero de ellos es ilustrar cómo se aborda la cuestión desde dos perspectivas cercanas aunque diferentes, la psicológica y la neurocientífica. El segundo de ellos consiste en ver cómo puede llegar a afirmarse injustificadamente bien la existencia del libre albedrío o bien su inexistencia sin ofrecer una demostración empírica en torno al cumplimiento o incumplimiento de las condiciones de posibilidad del mismo. 


\section{CRÍtICA A LA PERSPECTIVA PSICOLÓGICA: LIBERTAD COMO LIBRE-DE Y LIBRE-PARA}

Muchos son los autores que han atendido a la cuestión de la libertad desde una perspectiva naturalista, incluso antes del auge neurocientífico. Antes de profundizar en los problemas que la neurociencia encuentra a la hora de abordar la cuestión del libre albedrío conviene atender a los problemas con los que se ha enfrentado la psicología. Para ilustrar la insuficiencia de esta rama del saber en torno al problema del libre albedrío atenderemos a Aranguren, concretamente al capítulo de su Ética titulado «La realidad constitutivamente moral del hombre: moral como estructura». Entre las estructuras psicobiológicas que este autor analiza, y que caracterizarían al ser humano como un ser estructuralmente moral, se encuentra la estructura de la libertad, a la que caracteriza en dos dimensiones. Atender a su propuesta permitirá comprender la dimensión psicológica más básica de la libertad, poniendo de relieve las dificultades que esta perspectiva encuentra para resolver el problema de si la conducta humana es libre o está determinada unívocamente. Tras ello profundizaremos en la cuestión dando paso a una crítica del mismo tipo, esta vez enfocada a las neurociencias.

Según propone Aranguren, las estructuras psicobiológicas de nuestra especie configuran la libertad en dos dimensiones, libertad como libre-de estímulos, en tanto que el ser humano escapa del esquema conductual estímulo-respuesta, y libertad como libre-para, en tanto que el esquema estímulo-cognición-respuesta abre, al menos aparentemente, un abanico de posibles respuestas ante un mismo estímulo.

«Efectivamente, la libertad para preferir esta o la otra posibilidad procede de que la inteligencia como versión a la realidad concibe o proyecta diversas posibilidades de salida de la situación actual.» ${ }^{10}$

A lo cual añade que se trata "de una estructura a la vez compleja y unitaria», siendo el caso que «la libertad no reposa sobre sí misma, sino que se levanta sobre las estructuras psicobiológicas» ${ }^{11}$.

«Pero el organismo humano, a fuerza de complicación y formalización, no puede ya dar, en todos los casos, por sí mismo, respuesta adecuada o ajustada, y queda así en suspenso ante los estímulos, "libre-de" ellos (primera dimensión de la libertad). Las estructuras somáticas exigen así, para la viabilidad de este hiperformalizado ser vivo, la aparición de la inteligencia. ¿Qué significa aquí, primariamente, la palabra "inteligencia"? Pura y simplemente que el hombre, para subsistir biológicamente, necesita "hacerse cargo" de la situación, habérselas (concepto de «habitud») con las cosas - y consigo mismo- como "realidad" y no meramente como estímulos.» ${ }^{12}$

El ser humano, caracterizado así, ante un estímulo no tiene una respuesta predeterminada por su biología, en ese sentido es libre-de los estímulos. Para

\footnotetext{
10 Aranguren, J. L. L., Ética, Alianza Editorial, Madrid, 1994, p. 51.

11 Ibíd., p. 52.

12 Aranguren, J. L. L., o.c. p. 47.
} 
configurar una respuesta ha de hacer uso de la inteligencia. Existen, pues, unas tendencias - $\mathrm{O}$ «ferencias» en terminología del autor- inconclusas, en forma de predisposiciones biológicas que orientan la acción probabilísticamente, y no deterministamente ${ }^{13}$. Será la cognición la que complete el proceso de respuesta al medio, en el esquema conductual estímulo-cognición-respuesta. La elección entre posibilidades pone en ejercicio la otra dimensión de la libertad, la libertadpara preferir una acción y no otra ${ }^{14}$.

Ahora bien, la propuesta de Aranguren en relación a esta cuestión no resuelve, sin embargo, el problema de si existe o no el libre albedrío. Bien podría suceder que, a pesar de que el ser humano en su conducta es libre-de estímulos y libre-para realizar diferentes acciones mediando con la cognición, los procesos cognitivos en interrelación con los elementos estimulantes configurasen una red de causalidad que determinase la conducta, estando la cognición sujeta a una cadena causal, y siendo la elección realizada, entre las diferentes posibilidades aparentes, la única respuesta posible en unas condiciones dadas. En tal caso la libertad existiría solo en apariencia, ser libre-de estímulos tal como plantea Aranguren significaría que los estímulos ambientales no serían los únicos que determinarían la respuesta unívoca, sino que además habría factores cognitivos que se sumarían a la determinación conductual, sin evitar así su univocidad, y sin escapar, por tanto, de la cadena causal. Ser libre-para significaría, a su vez, que existirían virtualmente varias posibles respuestas ante ciertos estímulos ambientales, pero no existiría la posibilidad real de dar una respuesta diferente a la que de hecho se da, pues los factores cognitivos sumados a los ambientales determinarían una respuesta también unívoca y causal.

Si bien hemos atendido a Aranguren por ofrecer un ejemplo paradigmático de la perspectiva psicológica en relación a la cuestión del libre albedrío, podemos afirmar que la psicología, en términos más generales, se presenta igual de infructuosa a la hora de desvelar la existencia o inexistencia de las condiciones de posibilidad de la libertad. Aranguren acierta al señalar, aunque implícitamente, las mismas condiciones que hemos señalado anteriormente, coincidiendo así con Cortina y Evers. Respecto a la condición $L 1$, que refiere a la posibilidad de optar entre más de una opción a la hora de actuar, Aranguren atiende a ella al hablar de libertad-de, caracterizando al sujeto como libre-de univocidad, es decir, capaz de escoger entre más de una opción. En cuanto a L2, referente a la autodeterminación, Aranguren atiende a ella al hablar de libertadpara, caracterizando al sujeto como libre-para escoger por autodeterminación. Lamentablemente su propuesta no logra demostrar que estas condiciones

13 En relación a esta cuestión cabe mencionar la idea de la moralidad estratificada en dos niveles, el de las estructuras biológicas, que son orientativas y compartidas por todos los miembros de la especie, y el de los contenidos, que corresponden al bagaje cultural adquirido a partir de las diferentes respuestas posibles ante un mismo estímulo.

Ayala, F. J. y Cela Conde, C. J., «La filogénesis de la moral», en: Ayala, F. J. y Cela Conde, C. J., Senderos de la evolución humana, Alianza Editorial, Madrid, 2001, p. 533.

14 Aranguren, J. L. L., o.c. p. 48. 
.de hecho se den, ni tampoco los presupuestos sobre los que se asientan. En cuanto a $L 1$ Aranguren da por sentado que al escapar del esquema estímulorespuesta existe la posibilidad real de ofrecer diferentes respuestas ante las mismas causas, sin tener en cuenta que los factores cognitivos del esquema estímulo-cognición-respuesta pueden operar deterministamente haciendo que la conducta sea en todo caso unívoca y causal. En cuanto a L2 Aranguren da por supuesto que en el proceso cognitivo que articula la respuesta opera un yo que permite que la decisión y la acción se den por autodeterminación, sin embargo, no demuestra la existencia de ese yo ni de su capacidad de autodeterminación.

\section{CRÍtica A LA PRETENSIÓN NEUROCIENTÍFICA DE CUANTIFICAR LA LIBERTAD}

Tomando el relevo a la psicología algunos neurocientíficos se han propuesto resolver el problema desde su campo de investigación. Cabe preguntarse, no obstante, si tal cosa es posible. El determinismo neurocientífico nace de manos de los experimentos de Konhuber y Deecke ${ }^{15}$, pero será Libet quien abra un debate polémico en torno al tema. Tratando de apoyar la tesis de que existe el libre albedrío sus experimentos neurocientíficos sugirieron lo contrario. Su conocido experimento trata de medir si la decisión consciente es anterior o posterior a la actividad cerebral que posibilita su ejecución. Se pretendía mostrar que la decisión consciente de realizar una determinada acción vendría dada antes que el proceso neuronal de poner en marcha esa acción. Sin embargo, el experimento identifica una carga eléctrica que aparece con anterioridad a la decisión consciente, precediéndola en unos milisegundos. Lo que el autor concluye es que esa carga, a la que llama potencial de disposición, impulsa y en cierta medida determina la decisión con anterioridad a que esta aparezca en la conciencia. Por lo tanto, el proceso de la voluntad se iniciaría inconscientemente, unos milisegundos después tendríamos conciencia de él. No cabría hablar de acciones libres en la medida en que el sujeto no las elige conscientemente. Pudo matizar más tarde el resultado de sus experimentos, sugiriendo que, a pesar de que la decisión parte de forma inconsciente desde el cerebro, la voluntad puede vetarla, existiendo así cierto terreno para la libertad y la responsabilidad en la acción. ${ }^{16}$

15 Kornhuber, H. H., y DeEcKe, L., «Hirnpotentialanderungen bei Willkurbewegungen und passiven Bewegungen den Menschen: Bereitschaftspotential und reafferente Potentiale», en: Pflugers Archiv fur die gesamte Physiologie des Menschen und der Tiere, 284(1) (1965) 1-17.

KornhuBER, H. H. et al. (1989). "Will, volitional action, attention and cerebral potentials in man: Bereitschaftspotential, performance-related potentials, directed attention potential, EEG spectrum changes», en W. A. Hershberger (Ed.), Volitional action, Elsevier, Amsterdam, 1989, pp. 107-168.

16 LiBET, B. et al., «Time of conscious intention to act in relation to onset of cerebral activity (readiness potential)», Brain, 1983, 106: 623-642. 
La propuesta de Libet ha generado todo tipo de reacciones, entre las cuáles podemos diferenciar dos grandes grupos. Por un lado, existen quienes ven en su propuesta una confirmación de que la conciencia es una manifestación del cerebro, siendo una ilusión nuestra autopercepción como sujetos libres. Ligada a esta postura encontramos aquella que considera al cerebro como un mecanismo más de un universo determinista, sin escapar de la univocidad que esto supone, no habiendo por tanto espacio para la libertad. Por otro lado, sin embargo, se han producido una serie de reacciones que critican las conclusiones del experimento, considerándolas desproporcionadas en relación con lo que en realidad éste puede demostrar empíricamente. Cabe atender a estas críticas para identificar algunos apuntes metodológicos relevantes y para poner en tela de juicio la posibilidad misma de resolver el problema desde una perspectiva naturalista.

Lo que Libet propone es muy sugerente, pero las conclusiones del experimento, tal y como argumentan sus críticos, no son adecuadas partiendo de los datos empíricos obtenidos gracias al mismo. Su experimento y las numerosas críticas que ha recibido ${ }^{17}$ ponen sobre la mesa el problema, cada vez más acuciante, de aclarar cómo se ha de proceder a la hora de investigar el problema del libre albedrío desde el paradigma naturalista de las ciencias empíricas. Si la ciencia pretende abordar este tema hemos de preguntarnos cómo debe hacerlo y si está capacitada para resolver un problema de esta envergadura. Para esbozar una respuesta a esta pregunta señalaremos los puntos más relevantes que Cortina plantea, quien recoge a su vez las críticas que Habermas dirige contra las conclusiones del experimento. Estos puntos son los siguientes:

- El experimento plantea una situación artificial y unos presupuestos que hacen imposible su extensión a condiciones más generales de toma de decisiones.

- Nuestras decisiones siempre cuentan con una parte de planificación, no pueden medirse por instantes como Libet plantea. Y por si faltara poco,

LiBET, B., "Cerebral processes that distinguish conscious experience from unconscious mental functions», en: Eccles J. C. y CReutzfeldt O. (eds.), The principles of design and operation of the brain, Pontificiae Academiae Scientiarum Scripta Varia, Rome, Vatican, 78, pp. 186-202.

Libet, B., «Do We Have Free Will?» en: Journal of Consciousness Studies, Vol. 6, № 8-9, (1999) 47-57(11).

17 Algunas de ellas son:

CORTINA, A., O. c., 164-172.

Habermas, J., Entre naturalismo y religión, Paidós, Barcelona, 2006, 162-164.

Pallarés, D., "My brain made me not do it: an emergentist interprestation of Benjamin Libet», en: Ramon Llull Journal of Applied Ethics, issue 7 (2016) 121-141.

Roskies A. L (2010), "Why Libet's studies don't pose a threat to free will», en Conscious Will and Responsibility, ed. Sinnott-Armstrong, W. y Nadel, L., Oxford University Press, New York, pp. 11-22. 
los sujetos del experimento habían sido entrenados, pudiendo generar disposiciones mecánicas en el cerebro a esas decisiones.

- Libet supone la existencia de un yo, en algún lugar del cerebro, que no se ha podido demostrar empíricamente (y que es uno de los presupuestos $[L 2 a]$ de las condiciones de posibilidad del libre albedrío que hemos indicado en apartados anteriores).

- La decisión libre quizás no tenga que ser necesariamente consciente, como Libet propone, quizás baste con que sea virtualmente consciente. Podría actuarse de forma no consciente, pero porque se quiere hacer, y después poder argumentar conscientemente el porqué de esa decisión.

- Le falta conexión interna con razones, las cuales son necesarias para que exista una deliberación que permita la libertad.

- Además, sostiene Cortina, es imposible decir desde la ciencia empírica que la libertad no exista, el método empírico solo puede tratar hechos que puedan experimentarse, afirmar que todo está determinado causalmente es una afirmación metafísica que escapa al alcance de cualquier ciencia.

Un análisis detallado de estos puntos permite configurar un listado de recomendaciones metodológicas que han de regir la investigación neurocientífica en torno al libre albedrío:

- Las condiciones de todo experimento son locales y artificiales, contando con unos presupuestos que imposibilitan la extensión de los resultados a contextos más amplios de toma de decisiones. Esto supone que los resultados de un experimento han de ser manejados con cautela y no extender injustificadamente las conclusiones a contextos de decisión diferentes a los del experimento.

- Por el momento no existen criterios adecuados para la cuantificación de las decisiones. El proceso de decisión es amplio y complejo, de ahí que una de las críticas a Libet sea su pretensión de cuantificar la decisión en instantes. Encaja aquí también la crítica a Libet de no incluir las razones en la toma de decisiones en su experimento. El complejo proceso de la decisión se ve intervenido por las razones que llevan a tomar una y no otra decisión.

- El entrenamiento y la generación de disposiciones mecánicas son, entre otros, algunos factores que pueden adulterar el resultado de un experimento basado en la toma de decisiones. Hay que tener en cuenta estos factores y diseñar el experimento de forma pertinente.

- La investigación neurocientífica en torno a la libertad cuenta con algunos presupuestos que no han podido demostrarse empíricamente. Un ejemplo de ello es la existencia de un yo, que sería el decisor libre, en caso de haber libertad.

- Es necesario aclarar conceptos básicos, como el de decisión libre. Mientras que Libet considera que la decisión libre ha de ser consciente, otros autores no lo consideran así. 
- Que haya o no libertad es una realidad, pero no toda realidad es necesariamente cognoscible desde las ciencias empíricas. Encontramos aquí el problema de si las ciencias están capacitadas para ofrecer algún conocimiento sobre la existencia o no existencia de la libertad, o si, por el contrario, no son capaces de ello.

Libet no logra, y en un sentido más general tampoco la neurociencia, resolver si las condiciones de posibilidad del libre albedrío existen en el ser humano. Respecto a L1 (la posibilidad real de escoger entre dos cursos de acción diferentes) el experimento no ofrece datos suficientes para concluir que la conducta se produzca de forma unívoca en el marco de una cadena causal, sin embargo, tal cosa parece darse por sentado al considerar que el potencial de disposición funciona deterministamente, ya que se produce antes que la aparición de la decisión en la conciencia. La posibilidad de que exista una contingencia causal o la posibilidad de que pueda decidirse de forma libre sin ser consciente son, entre otros, algunos escenarios que no deberían ser descartados. En cuanto a L2 (la elección se ha de dar por autodeterminación) Libet tampoco logra demostrar que esta condición no se dé, pues no puede demostrar ni desmentir la existencia de un yo, que sería el decisor libre.

Esta infecundidad de la neurociencia en torno al problema del libre albedrío nos remite a reconsiderar el último de los puntos expuestos anteriormente. Este tiene especial relevancia ya que eleva la crítica no solo a cómo operar metodológicamente, sino a la suficiencia misma del método científico para resolver el problema del libre albedrío. Si, como Cortina argumenta, la ciencia empírica no está capacitada para ofrecer un conocimiento empírico sobre la existencia o inexistencia del libre albedrío, entonces las recomendaciones metodológicas expuestas anteriormente serían vacuas, pues el mismo intento de conocer mediante las ciencias naturales si somos o no libres sería una tentativa condenada al fracaso.

Los problemas se plantean, en principio, por una cuestión de método. Quien desee recurrir a lo que ha venido a llamarse «método científico», propio de las ciencias naturales, tiene que investigar causalmente, [...]. Lo que no cabe en esa relación causal de acontecimientos observables, expresados en tercera persona, es o bien lo científicamente incognoscible o bien lo inexistente.

Reconocer que la libertad no puede ser conocida por este método es practicar algo así como un agnosticismo de la libertad, que es lo que profesó Kant en la Crítica de la razón pura: no podemos probar objetivamente la existencia de la libertad porque, si existe, no es el método empírico de las ciencias el que nos permite llegar a ella. Sin embargo, lo que no puede hacer el científico es afirmar que la libertad no existe porque no puede probar su existencia haciendo uso de su método. Una afirmación semejante es metafísica, no científica. El neurocientífico no puede permitirse un ateísmo de la libertad..$^{18}$

18 CORTINA, A., o.c. pp. 187-188. 
Más allá de la necesidad de establecer unos procedimientos metodológicos adecuados, Habermas, coincidiendo en esto con Cortina, considera la posibilidad de que la existencia o inexistencia del libre albedrío no sea algo medible por las ciencias empíricas. Sobre esta cuestión nos advierte de que algunas cosas son, simplemente, incuantificables.

Como en todo design, también aquí surge la pregunta sobre qué es lo que se mide, además de la cuestión filosófica de entrada concerniente a qué cosas pueden ser sometidas a medición. ${ }^{19}$

Considerando que pueda haber una imposibilidad de las ciencias empíricas para resolver la pregunta de si somos o no libres, Cortina sugiere que abordemos la cuestión de forma más asequible, hablando de condiciones de la libertad, y no de causas. Lo que nuestra autora propone, por tanto, es una sustitución de conceptos. Mientras que el concepto de causa es entendido en términos de necesidad, el concepto de condición tiene un carácter probabilístico. Esto permitiría evitar los problemas del método científico asociado al concepto de causa. La acción humana se ve siempre condicionada por una serie de factores, aun si guarda cierto espacio para la libertad, hablar de condiciones contempla estos factores, tomados como condicionamientos que limitan la conducta de forma probabilística, y evita a su vez los problemas derivados de hablar de la acción en términos de necesidad.

La cuestión de si somos o no libres sigue siendo hoy en día una pregunta sin respuesta. En primer lugar la ciencia empírica sugiere que quizás sea posible resolver el problema a través de su método. Por ello, mientras los científicos mantengan su interés en torno a la cuestión, se ha de alentar una investigación que sea adecuada metodológicamente, solo de esta manera podemos esperar que cualquiera de los pasos que se den desde las ciencias empíricas se realicen en la dirección adecuada y sin excederse en sus competencias. Quizás una adecuada investigación científica pueda en el futuro arrojar algo de luz sobre esta cuestión tan problemática a día de hoy. Por otro lado, tal y como hemos expuesto, existe la posibilidad de que la ciencia no sea capaz de resolver el problema, simplemente porque no esté capacitada para ello. Por este motivo se ha de mantener la labor de la filosofía, que sigue siendo necesaria para abordar la cuestión de la libertad.

\section{CONCLUSIONES}

La existencia de la libertad, entendida como libre albedrío, depende de que las estructuras psicobiológicas de nuestra especie permitan las condiciones de posibilidad de la misma, que son: L1) dadas unas condiciones ha de existir la posibilidad real de escoger entre dos cursos de acción diferentes, y L2) la elección

19 Habermas, J., Entre naturalismo y religión, Paidós, Barcelona, 2006, p. 162. 
se ha de dar por autodeterminación, no por azar ni por indeterminación. Estas condiciones implican una serie de presupuestos que toda investigación científica debería de resolver, siendo estos, en realidad, cuatro condiciones de posibilidad que resultan al desglosar las dos citadas anteriormente. Entre los presupuestos de $L 1$ se encuentran: L1a) Existencia bien de una fisura en la cadena causal, o bien de una bifurcación posible en la misma (contingencia causal); L1b) existencia de la posibilidad, brindada por el cerebro, bien de generar una causa que produzca una decisión y una acción (el yo sería esta causa, ver $L 2 b$ ), bien de dirigir la conducta hacia una de las opciones existentes en una contingencia causal. Entre los presupuestos de L2 encontramos los siguientes: L2a) Existe un yo, que sería el decisor y el actor; L2b) el yo tiene la capacidad de operar como causa en la decisión y en la acción, bien como una nueva causa en una fisura en la cadena causal, bien como causa de la elección entre las opciones posibles en una contingencia causal.

En el caso de que tales condiciones no se den, se habría de considerar la posibilidad de la libertad como una ilusión, siendo una autopercepción resultante de nuestras facultades cognitivas. Resolver si de hecho estas condiciones se dan o no en nuestra especie es una cuestión que se ha erigido como uno de los ejes de investigación en la neurociencia, extendiéndose a su vez a la neuroética y al neuroderecho en la medida en que el libre albedrío es una de las condiciones de posibilidad de lo moral, pues una conducta genuinamente moral precisa que el agente pueda actuar con libertad, solo así conservarían su sentido conceptos morales como el de responsabilidad, obligación y deber, entre otros.

La comprensión del libre albedrío como una estructura psicobiológica implica que la existencia o inexistencia de este sea una realidad, no obstante esto no significa que dicha realidad pueda ser cuantificada o medida desde el paradigma naturalista de las ciencias empíricas. Ni la psicología ni la neurociencia han logrado resolver si tales estructuras de hecho existen.

Desde la psicología la libertad puede plantearse estructuralmente en dos dimensiones, libertad-de y libertad-para, referentes a que el sujeto pueda hallarse libre-de estímulos y libre-para diferentes respuestas. Esta doble dimensión refiere a las condiciones L1 (posibilidad de actuar de diferentes modos ante unas mismas causas) y $L 2$ (autodeterminación), respectivamente. Esta caracterización sin embargo no garantiza que exista la libertad como libre albedrío. Respecto a $L 1$ existe la posibilidad de que la cognición que media entre el estímulo y la respuesta haga que esta última no sea unívoca ante un determinado estímulo externo, pero sin que esto signifique que los procesos cognitivos implicados en la configuración de la respuesta no sean determinantes o que la conducta no se dé por indeterminación. Respecto a L2 no se logra demostrar la existencia o inexistencia del yo decisor ni de su capacidad para actuar por autodeterminación.

Entre las hipótesis que algunos autores plantean para considerar viable la existencia del libre albedrío hemos mencionado el concepto de causalidad contingente, referido a la posibilidad de que exista una causalidad compatible con la libertad de elección, este concepto plantea que ante una misma causa 
pueden darse dos consecuencias diferentes, entre las cuales la elección sería posible. Asimismo, en aras de mantener vigente el concepto de responsabilidad hemos mencionado la idea de una responsabilidad no consciente, en función de la cual se puede ser responsable a pesar de los procesos no conscientes que subyacen a las decisiones y a las acciones, ya que somos personalmente responsables de esos estados y procesos en la medida en que podemos influir en ellos.

Entre las tentativas neurocientíficas de cuantificar la libertad como hecho neurobiológico el experimento de Libet se sitúa como uno de los ejemplos más celebres y paradigmáticos, aunque también muy problemático, por ello lo hemos tomado como eje en torno al cual articular una crítica aplicable a cualquier pretensión de investigación neurocientífica y naturalista del libre albedrío. En relación a ello cabe hacer las siguientes recomendaciones metodológicas:

- Experimentación: Un experimento plantea una situación artificial, hay que ser cautos si se pretende extender el resultado a condiciones más generales de decisión.

- Diseño del experimento: Existe el riesgo de adulterar el resultado del experimento si no se diseña adecuadamente. Por ejemplo el entrenamiento puede generar disposiciones mecánicas que modifiquen las condiciones de la decisión. Hay que diseñar el experimento de forma adecuada.

- Aclarar terminología: Diferentes investigadores presentan diferentes concepciones de los mismos elementos, es necesaria una aclaración de términos. Por ejemplo es necesario especificar en qué consisten conceptos como el de decisión libre, que para algunos autores ha de ser consciente mientras que para otros no necesariamente.

- Criterios de cuantificación: Es necesario aclarar los criterios de cuantificación de los elementos abordados en la investigación. Por ejemplo, Libet pretende cuantificar la decisión en instantes, mientras que otros autores someten a crítica esta pretensión.

- Presupuestos de la investigación: Han de ser especificados. Uno de los presupuestos es la existencia de un yo, que no ha podido ser identificado mediante investigación neurocientífica, y que sería el decisor libre.

Libet, y la neurociencia en general, no logra demostrar la existencia o inexistencia de las condiciones de posibilidad del libre albedrío. Respecto a L1 la cuantificación de la actividad cerebral, al menos a día de hoy, no permite afirmar concluyentemente que la conducta humana opere deterministamente. Respecto a $L 2$ no se ha podido identificar empíricamente la existencia de un yo, ni tampoco demostrar su inexistencia, y por consiguiente tampoco se ha podido demostrar su capacidad para actuar por autodeterminación.

Además de estas recomendaciones metodológicas, que pretenden guiar la investigación, y ante la infecundidad de las neurociencias en su propósito de ofrecer una respuesta al debate determinismo-libertad, se plantea también la cuestión de si las ciencias empíricas son incapaces de resolver este problema, 
considerando la posibilidad de que la libertad no pueda ser sometida a medición desde el método científico y el paradigma naturalista. En relación a ello cabe hacer las siguientes indicaciones:

- Agnosticismo como posibilidad: El neurocientífico ha de tomar en consideración la posibilidad de que la existencia o inexistencia del libre albedrío sea una realidad incognoscible para las ciencias empíricas, por no poder someterse a medición a través de su método.

- Importancia de la filosofía: La tarea de la filosofía es necesaria y relevante a la hora de abordar y pensar la cuestión de la libertad, algo que se acentúa ante la posibilidad del agnosticismo de las ciencias empíricas respecto a esta cuestión.

- Condiciones, no causas: La dificultad que supone tratar el tema de la libertad desde el paradigma de causalidad propio de las ciencias empíricas puede evitarse sustituyendo el concepto de causa por el de condición, aquel supone hablar en términos de necesidad, éste supone hablar en términos de probabilidad, reconociendo los factores que imponen límites a la acción.

BiBLIOGRAFÍA

Aranguren, J. L. L. (1994). Ética. Madrid: Alianza Editorial.

Ayala, F. J. (2006). «Las raíces biológicas de la moral», en: Ayala, F. J., La evolución de un evolucionista. Valencia: Universidad de Valencia, pp. 351-369.

Ayala, F. J. y Cela Conde, C. J. (2001). «La filogénesis de la moral», en: Ayala, F. J. y Cela Conde, C. J., Senderos de la evolución humana. Madrid: Alianza Editorial, pp. 517538.

Banks W. P. y Pockett S. (2007). «Benjamin Libet's work on the neuroscience of free will», en Blackwell Companion to Consciousness, ed. Velmans, S. y Schinder, S., Malden.MA: Blackwell, pp. 657-670.

Cortina A. (2007). Ética mínima: Introducción a la filosofía práctica. Madrid: Tecnos.

Cortina, A. (2012). Neuroética y neuropolítica: Sugerencias para la educación moral. Madrid: Tecnos.

Cortina, A. (ed.) (2012). Guía Comares de Neurofilosofía Práctica. Granada: Editorial Comares.

Evers, K. (2010). Neuroética. Cuando la materia se despierta. Madrid: Katz Editores.

Farah, M. J. (2012). "Neuroethics: The Ethical, Legal, and Societal Impact of Neuroscience» en: Annual Review of Psychology, 63, pp. 571-91.

Gould, S. J. y Lewontin, R. C. (1979). «The Spandrels of San Marco and the Panglossian Paradigm: A Critique of the Adaptationist Programme», The Royal Society of London, Series B, Vol. 205, No. 1161, 581-598.

Gould, S. J. y Vrba, E. S. (1982). «Exaptation - A Missing Term in The Science of Form», en Paleobiology, Vol. 8, $\mathrm{N}^{\circ}$ 1, 4-15.

Habermas, J. (2006). Entre naturalismo y religión. Barcelona: Paidós.

Illes, J., y Sahakian, B. J. (2011), The Oxford Handbook of Neuroethics. Oxford: Oxford University Press. 
Kant, I. (2000). Crítica de la razón práctica. Madrid: Alianza.

Kornhuber, H. H., y Deecke, L. (1965). «Hirnpotentialanderungen bei Willkurbewegungen und passiven Bewegungen den Menschen: Bereitschaftspotential und reafferente Potentiale», en: Pflugers Archiv fur die gesamte Physiologie des Menschen und der Tiere, 284(1), 1-17.

Kornhuber, H. H. et al. (1989). «Will, volitional action, attention and cerebral potentials in man: Bereitschaftspotential, performance-related potentials, directed attention potential, EEG spectrum changes», en W. A. Hershberger (Ed.), Volitional action. Amsterdam: Elsevier, pp. 107-168.

Libet, B. et al. (1983). «Time of conscious intention to act in relation to onset of cerebral activity (readiness potential)», Brain, 106: 623-642.

Libet, B., "Cerebral processes that distinguish conscious experience from unconscious mental functions», en: Eccles J. C. y Creutzfeldt O. (eds.), The principles of design and operation of the brain, Pontificiae Academiae Scientiarum Scripta Varia, Rome, Vatican, 78, pp. 186-202.

Libet, B. (1999). «Do We Have Free Will?» en: Journal of Consciousness Studies, Vol. 6 , $\mathrm{N}^{\mathrm{o}} 8-9$, pp. 47-57(11).

Pallarés, D. (2016). «My brain made me not do it: an emergentist interprestation of Benjamin Libet», en: Ramon Llull Journal of Applied Ethics, issue 7, pp. 121-141.

Roskies, A. L. (2010). «How Does Neuroscience Affect Our Conception of Volition?» en: Annual Review of Neuroscience, 33, pp. 109-130.

Roskies, A. L (2010), «Why Libet's studies don't pose a threat to free will», en Conscious Will and Responsibility, ed. Sinnott-Armstrong, W. y Nadel, L. New York: Oxford University Press, pp. 11-22.

Departamento de Filosofía, Universidad de Valencia

AndRÉs D. Richart Piqueras

Andres.Richart@uv.es

[Artículo aprobado para publicación en diciembre de 2016] 